\title{
Prendre le terrain pour objet : une expérience de recherche collective sur les espaces publics à Ibadan, Nigeria
}

Pauline Verger, Manon Le Bon, Mathilde Beaufils, Chloé Vincent et Justine Vignères

\section{(2) OpenEdition}

Journals

Édition électronique

URL : https://journals.openedition.org/cdg/6327

DOI : $10.4000 /$ cdg.6327

ISSN : 2107-7266

Éditeur

UMR 245 - CESSMA

Référence électronique

Pauline Verger, Manon Le Bon, Mathilde Beaufils, Chloé Vincent et Justine Vignères, « Prendre le terrain pour objet : une expérience de recherche collective sur les espaces publics à Ibadan, Nigeria », Carnets de géographes [En ligne], 14 | 2020, mis en ligne le 20 décembre 2020, consulté le 28 juin 2022. URL : http://journals.openedition.org/cdg/6327 ; DOI : https://doi.org/10.4000/cdg.6327

Ce document a été généré automatiquement le 21 mai 2021.

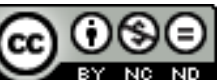

La revue Carnets de géographes est mise à disposition selon les termes de la Licence Creative Commons Attribution - Pas d'Utilisation Commerciale - Pas de Modification 4.0 International. 


\title{
Prendre le terrain pour objet : une expérience de recherche collective sur les espaces publics à Ibadan, Nigeria
}

\author{
Pauline Verger, Manon Le Bon, Mathilde Beaufils, Chloé Vincent et Justine
} Vignères

1 Les autrices de cet article sont cinq étudiantes de l'École Normale Supérieure (ENS) qui ont eu l'opportunité de participer, avec dix étudiant·e's nigérian'e-s, à une masterclass portant sur «les espaces publics dans les villes africaines» organisée du 19 au 28 février 2019 par le département Géographie et Territoires de l'ENS et l'Institut français de Recherche en Afrique du Nigeria (IFRA) ${ }^{1}$. Étudiantes en géographie, sociologie politique, urbanisme et histoire de l'art, nos intérêts académiques et professionnels différents ont trouvé écho dans les divers questionnements pluridisciplinaires soulevés au cours de cette masterclass ${ }^{2}$. Après trois jours de présentations théoriques et méthodologiques organisés avec Unilag, l'université de Lagos, nous sommes parties à Ibadan pour cinq jours de mise en application de ces acquis théoriques et méthodologiques afin de réfléchir ensemble à l'applicabilité de la notion « d'espace public » dans le contexte nigérian.

2 Le terme «terrain » convoque les idées de délimitation, de déplacement, d'entrée et de sortie sur un territoire souvent étranger et nouveau. Les méthodes ethnographiques et la dénomination même de terrain sont d'abord formulées pour l'analyse des espaces lointains par des chercheur:se's occidentauxales et véhiculent toujours une certaine exotisation des Suds (Hancock, 2007) ${ }^{3}$. Les stages de terrains jouent également un rôle prépondérant dans la formation des étudiante's en sciences sociales, en particulier dans le cadre de la formation de future's enseignante-s chercheur-se's (Zrinscak, 2010) ${ }^{4}$. A ce titre, ils constituent un rite de passage permettant de saisir les implicites du métier, aussi bien les questionnements éthiques qui entourent la pratique du terrain 
que les conditions matérielles de réalisation de ces mêmes terrains (Vergnaud, Le Gall, 2017).

3 A l'issue d'une masterclass théorique et pratique sur les divers régimes de publicité des espaces (Staeheli, Mitchell, 2007) à Ibadan au Nigéria, nous nous sommes interrogées sur nos pratiques de terrain en étudiant les quatre espaces suivants: Agodi Gate, une importante gare routière et son marché des pièces détachées pour automobiles; Oje Market, l'un des principaux marchés de la ville ; le rond-point situé entre l'hôtel de ville d'Ibadan, Mapo Hall et Beere, lieu de transit et de commerce que nous désignerons dans le reste de l'article par le terme rond-point de Beere; et Cocoa Mall et Heritage Mall, regroupés en un même centre commercial, dans le quartier d'affaires de Dugbe. Dans les faits, le terrain, entendu comme "la pratique empirique de collecte de données in situ " (Volvey, Calbérac, Houssay-Holzschuch, 2012: 442), devait nous permettre d'apprendre à mobiliser diverses méthodes quantitatives et qualitatives (statistiques sur les fréquentations du mall, questionnaires, capture de paysages sonores, diverses méthodes de cartographie participative, etc.), qui peuvent être utilisées par l'ensemble des sciences humaines et sociales ${ }^{5}$.

Figure 1. Localisation des quatre terrains étudiés à Ibadan

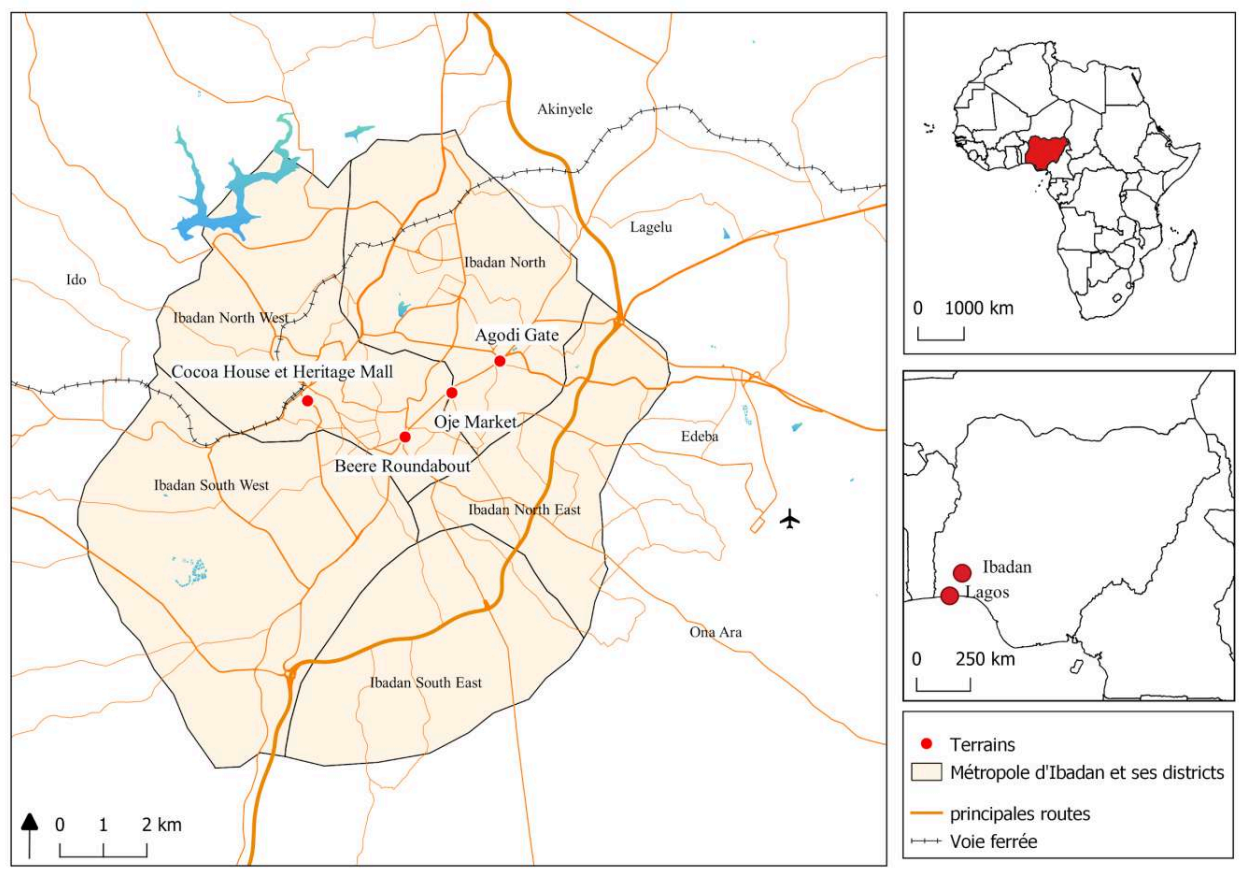

Source : fonds de carte OpenStreetMap ; réalisation : Autrices, 2019

4 Cette initiation au travail de terrain fut pour la majeure partie d'entre nous une première expérience de recherche dans une ville d'Afrique sub-saharienne. Cela nous a permis de nous confronter à certains préjugés (les nôtres, ceux des étudiant·e's nigérian'e's, ceux des habitant·e's, ceux de nos assistante'es de recherche ${ }^{6}$ ou encore des encadrante-s). Ainsi, nous avons choisi dans cet article d'analyser nos diverses expériences de terrain afin de revenir sur ce que la pratique du terrain, c'est-à-dire à la fois l'entrée de jeunes étudiantes blanches occidentales dans ces espaces publics mais aussi l'activité de recherche (l'observation, la «flânerie » dans des espaces de circulation, etc.) montrent, à la fois de contraignant et de révélateur, des espaces 
étudiés et des méthodes utilisées. À partir de la remise en cause de la pratique du terrain en sciences sociales comme méthode objective et universaliste, opérée en partie par la géographie féministe (Rose, 1993), le «terrain» de la géographie a pu être transformé en objet épistémologique (Volvey, Calbérac, Houssay-Holzschuch, 2012). Dans cette optique, la généralisation de ces méthodes ethnographiques, dont la démarche réflexive est aujourd'hui l'une des composantes, peut être conçue comme une norme de validation scientifique dans le champ académique occidental, et de ce fait renforcer une division entre "monde occidental producteur de théorie et le reste du monde entendu plus ou moins comme un terrain » (Jazeel, cité in Dikeç, $2010: 803)^{7}$.

5 Au cours de notre travail en commun avec les étudiante's nigérian'e-s de la masterclass, il nous est apparu que l'utilisation de ces méthodes et la réflexivité allant de pair n'était pas valorisées de la même manière dans un cursus académique nigérian. Cette démarche apparaît donc relative à un champ académique contextuellement situé, imposant cependant ses critères à la production scientifique mondiale et $a$ fortiori africaine. Nous regrettons ainsi que cet article, écrit à dix mains, ne puisse également témoigner de l'expérience de recherche de nos camarades nigérian'e-s. La distance physique et linguistique n'a pas rendu cela possible pour cet article, mais l'écriture d'un article collaboratif en anglais pourrait permettre de pallier en partie cette dissymétrie.

Ainsi, ce retour réflexif nous paraît d'autant plus nécessaire que notre couleur de peau, notre genre, notre âge, notre nationalité et notre statut d'étudiante influaient sur ce que nous pouvions observer des terrains mais aussi sur le terrain lui-même (notamment du fait des interactions avec les usagere's de ces espaces que notre présence impliquait). L'analyse proposée est ponctuée de récits ethnographiques qui visent à expliciter nos postures et positionnements individuels et la manière dont ils ont pu orienter nos expériences de terrain (Dupont, 2014). Pour cela, nous suivons une démarche chronologique afin de mettre en récit nos entrées à la fois sur les terrains et dans nos groupes de recherche respectifs. Puis nous nous interrogeons sur ce que nos pratiques du terrain -entrées, négociations pour y rester, sorties- révèlent de ces espaces et de nos positionnements. Dans un dernier temps, nous développons la dimension non plus seulement spatiale mais aussi temporelle de ce terrain.

\section{DES TERRAINS À ENTRÉES MULTIPLES}

\section{L'ENTRÉE SUR LE(s) TERRAIN(s)}

7 Travailler sur les espaces publics à Ibadan nécessite de réfléchir au préalable à la pertinence de l'usage dans le contexte nigérian d'une notion multiple dans ses significations et largement débattue. La notion d'espace public recouvre une acception à la fois métaphorique et matérielle, résultant d'une construction historique occidentale (Navez-Bouchanine, 2001). Les espaces publics naissent de la coprésence d'individus anonymes. Ils peuvent être considérés comme des espaces où l'on peut se fondre dans la masse, des espaces parfois libérateurs (Cattan, Leroy, 2010), où l'on peut paradoxalement échapper au regard d'autrui. Pour nous, cet anonymat a été levé dès notre arrivée sur le terrain. D'abord par notre positionnement de chercheuses, puisque nous sommes amenées à nous présenter, à expliquer notre présence, voire à la justifier, pour mener à bien notre enquête. Ensuite, en tant que jeunes femmes blanches européennes, notre couleur de peau, notre âge et notre genre ont généré une situation 
de visibilité. En témoignent les fréquentes interpellations qui ponctuaient nos passages dans les espaces publics : «Oyibo, oyibo! $»^{8}$.

Nous avons pu tirer profit de cette situation de visibilité, dans la mesure où les usagere's des espaces publics étudiés venaient spontanément à notre rencontre et cherchaient à entamer la conversation avec nous. Toutefois, la présence à nos côtés d'assistante's de recherche, facilement identifiée's comme nos accompagnateur.rice's, a $\mathrm{pu}$ agir comme un filtre dans nos interactions avec les gens qui avaient tendance à s'adresser à nous indirectement, par leur entremise. Par ailleurs, les questions sur notre pays d'origine étaient un passage obligé dans nos entretiens. De manière générale, notre présence nous est apparue comme un élément perturbateur du fonctionnement des espaces publics étudiés. Citons à cet égard le spectacle de danse improvisé par un groupe de jeunes hommes pour attirer l'attention de l'une d'entre nous (Encadré 3). Notre présence modifiait en effet les interactions que l'on suppose habituelles entre les différente's acteur.rice's de l'espace, en concentrant l'attention autour de nous. La circulation s'en trouvait également changée, que ce soit par nos fréquents arrêts ou bien par nos allers-retours dans des moments d'observation, qui contrastaient avec la flânerie des clientees du mall, par exemple, ou encore les trajectoires des usageree's de la gare routière d'Agodi Gate. Enfin, notre statut d'observatrices, et non pas de consommatrices ou de flâneuses, a suscité de nombreuses interrogations des autres usagerees, en particulier dans le cadre d'espaces publics à forte dimension économique comme le marché et le mall.

Un élément perturbateur donc, mais aussi révélateur, en creux, du fonctionnement des espaces publics. Au mall par exemple, notre mobilité contrastait avec l'immobilité de la plupart des usagere's mettant en évidence le fait surprenant qu'ils/elles venaient moins pour consommer que pour passer le temps («chill and relax» d'après nos questionnaires). La question de la lisibilité de l'espace, de la compréhension de son organisation et de ses dynamiques, s'est imposée à nous au moment d'entrer sur le terrain. Il s'agissait de savoir s'orienter, s'y retrouver dans un contexte éloigné de nos représentations occidentales des espaces publics, depuis l'apparent désordre du marché des pièces détachées pour automobiles d'Agodi Gate, à la relative familiarité du mall. Ainsi, au mall, cette plus grande familiarité nous permettait d'avoir une certaine autonomie vis-à-vis de l'assistant de recherche dans nos déplacements et nos interactions, ce qui ne nous a pas empêchées d'avoir à franchir un certain nombre de seuils en vue d'entrer sur notre terrain (Encadré 1).

\section{Encadré $1^{9}$ : Le mall d'Ibadan, terrain singulier et pluriel aux multiples seuils}

Toutes les deux élèves en première année de l'ENS, et dernière année de Licence en Géographie, cette masterclass constituait respectivement notre premier et deuxième stage de terrain. Il a donc été décidé que nous serions exceptionnellement deux étudiantes françaises dans le même groupe de recherche au mall, un terrain jugé plus familier et sécurisant par nos encadrant·e·s.

Lors de notre préparation du terrain, nous avons systématiquement appréhendé le mall comme un espace unifié et homogène, et nous nous y référons d'ailleurs comme « le mall » au singulier. Or, la pratique du terrain fait d'emblée ressortir la pluralité de cet espace : à l'intérieur de l'enceinte du mall, se trouvent en effet Cocoa House, la tour de bureau; le restaurant Cocoa Dome; deux parkings, dont un 
souterrain ; le mall divisé en deux, Cocoa Mall et Heritage Mall, auxquels est accolé, comme un appendice indépendant, le supermarché de la chaîne sud-africaine Shoprite. Ces différents espaces sont délimités par des frontières plus ou moins visibles et matérialisées, et des filtres plus ou moins fins. Parmi les seuils très marqués, citons la grille d'enceinte, une barrière aussi bien physique que symbolique. Le poste de sécurité à l'entrée principale opère, si nécessaire, un deuxième filtrage des usager'e's jugée's indésirables ou n'appartenant pas au public du mall telle's que les vendeur'se's de rue ou les groupes d'enfants. En effet, en comparaison avec les autres espaces publics étudiés, le mall se distingue par la faible présence d'enfants. Ceux que nous croisons aux abords du mall guettent, depuis la grille, la sortie des client'e's pour mendier. Une autre opération de filtrage se déroule en parallèle, à l'entrée du parking souterrain sous la forme d'un péage informel (les péagers ne revêtent pas l'uniforme des employés et agents de sécurité). L'enseigne Shoprite, quant à elle, possède son propre service de sécurité, qui double le contrôle effectué à l'entrée principale d'une fouille systématique des sacs, à l'entrée et à la sortie du supermarché. À l'inverse, l'effet de seuil est beaucoup moins marqué à la limite entre les deux malls, visible seulement à quelques signes discrets comme la différence d'éclairage et de dallage, ou l'uniforme des gardien'ne's.

Franchir ces seuils est la première étape de notre recherche sur le terrain. Chaque matin, nous nous présentons aux agents de sécurité. Nous avons également pu rencontrer le gérant de Cocoa Mall et avoir eu accès au troisième étage, fermé d'ordinaire au public sous prétexte d'être réservé à l'administration. En réalité, la surface occupée par les bureaux est assez faible, et il semblerait plutôt que l'importance de la vacance commerciale, dans le mall en général, à cet étage en particulier, ait poussé la direction à en restreindre l'accès.

10 L'entrée sur le terrain s'est donc faite par le passage de seuils successifs qui pouvaient varier, en nombre et en nature, en fonction des terrains d'étude. A Oje Market, par exemple, l'accès s'est fait de manière progressive, depuis les espaces publics commerciaux jusqu'aux espaces privés résidentiels et avec un effet de filtre, tout le monde n'ayant pas accès à ces derniers. A chaque seuil, il a fallu (re)négocier son entrée.

\section{PRENDRE PLACE DANS LE gROUPE DE RECHERCHE}

11 Si l'entrée sur le terrain est révélatrice de notre positionnement racial, social et genré, ceci est tout aussi vrai en ce qui concerne les premières relations entretenues au sein d'un groupe de recherche. Pour comprendre la manière dont ces rapports sociaux s'inscrivent dans l'espace de production des savoirs sur l'Afrique sub-saharienne, il convient tout d'abord de préciser que la masterclass était organisée en collaboration avec l'IFRA Nigeria, institut public de recherche sous tutelle du Ministère français des Affaires Étrangères, et basé au sein de l'Institut d'Études Africaines de l'Université d'Ibadan. Au cours de notre deuxième semaine de mise en pratique des acquis théoriques et méthodologiques, nous étions divisée's en quatre groupes composés chacun d'au moins une assistante de recherche également étudiante originaire d'Ibadan, d'au moins deux étudiantees nigérian'e's de la masterclass, d'une ou deux 
étudiante-s française-s et d'un'e encadrante de recherche. Si la composition genrée des groupes était variable, notre couleur de peau, notre nationalité française et la constitution des groupes qui en a découlé nous ont toutes les cinq dans un premier temps positionnées à part dans nos groupes de recherche respectifs, qu'il se soit agi de nous considérer a priori comme plus compétentes sur des questions théoriques et méthodologiques ou moins compétentes dans la compréhension des situations de terrain. Par exemple, lors de la restitution collective de notre première journée de terrain, l'une de nous, étudiante de master, discutant avec un doctorant nigérian, s'est vu répondre qu'elle pouvait faire la première présentation puisque, elle, «elle savait comment faire ». Cette partition implicite des rôles entre étudiante's qui tendait à assigner les étudiantes françaises du côté du théorique et les étudiant·e-s nigérians du côté de l'empirique n'est pas le fait d'une simple différence de formation, ni même d'une diversité linguistique, puisque d'autres étudiant·e·s nigérian'e-s ne parlaient pas yoruba. Faire sa place dans un groupe de recherche ainsi constitué a ceci de difficile que cela met en jeu des relations de domination intersectionnelles (liées au genre, à la classe, à la nationalité, etc.) (Çıngı Kocadost, 2017 ; Crenshaw, 1991).

$\mathrm{Au}$ sein du groupe de recherche, le statut des assistantees de recherche pose également question (Encadré 2).

Encadré 2 : Récit des relations avec un assistant de recherche, première journée au rond-point de Beere

Élève en quatrième année de l'ENS, j'étudie la science politique (études africaines) et la géographie. Du fait de ma formation, j'ai porté une attention particulière aux rapports différenciés à et dans l'espace en fonction du genre, du statut social apparent et de l'âge des enquêtée's.

Le premier jour, alors que nous arrivons pour la première fois sur le rond-point de Beere, nous nous divisons pour faire un tour du quartier. L., l'assistant de recherche de mon groupe, a l'air inquiet et ne veut pas me laisser seule. La veille, au marché, je me souviens avoir été énervée par son côté directif. Je lui propose un arrangement : nous allons tous les deux de Beere au Mapo Hall, moi sur le côté gauche de la rue, lui du côté droit, chacun note ses observations pour pouvoir comparer ensuite. Puis arrivée's à Mapo Hall, nous nous rejoindrons. Cela semble lui convenir, il a l'air rassuré, si quelque chose arrive il pourra intervenir.

Montant la rue, je me rends compte effectivement, qu'une femme seule, a fortiori blanche, marchant dans la rue n'a rien à y faire. Les vendeur'se's me saluent et me hèlent pour que je les rejoigne. Je réponds aux salutations mais continue ma route. Deux hommes, jeunes, commencent à me suivre, j'effectue un léger arrêt, ils s'arrêtent. Effectivement, ils me suivent. Les femmes seules dans cette rue sont soit vendeuses, soit en train de rejoindre leur moyen de transport, je me rapproche de l'une d'entre elles qui se dirige vers un minibus. Le minibus arrêté, je m'arrête également, me penche pour remettre ma chaussure et laisser passer un peu de temps, les deux hommes me dépassent, se retournent lorsqu'ils se rendent compte que je n'ai pas continué ma route, mais ne s'arrêtent pas. Je reprends ma marche, regarde de l'autre côté de la route, L. s'est arrêté inquiet, je lui souris et rassurée's nous reprenons notre chemin. Arrivé à Mapo Hall, il me dit « You were followed by two men ». Je lui réponds : «I know, everything's fine ». « Ok, you seem to 
know how to deal with that $»^{10}$ me dit-il finalement. Une fois à Mapo Hall, nous partageons ce que nous avons pu observer : lui, la division commerciale de la rue, les activités ne sont pas les mêmes en haut et en bas de la rue, moi, la répartition genrée de l'espace de la route, de la rue et de la devanture des habitations. Nos observations sont complémentaires, nous avons bien fait de nous diviser.

Une fois à Mapo Hall, nous réussissons à entrer en nous adressant au gardien. L. me dit : « ask him anything, I'll translate ». Je lui réponds : « thank you for the translation, but if you have any questions, ask them too $»^{11}$ essayant maladroitement de rétablir une relation plus symétrique. Cette situation se répétera les jours suivant, L. semble se considérer véritablement comme mon assistant, nous sommes tou.te-s les deux étudiante's, cela me gêne tout le long de notre terrain.

Plusieurs fois, L. nous fait part de son appréhension quant à notre présence au rond-point de Beere. L. n'est pas simplement assistant de recherche, c'est un étudiant de l'Université d'Ibadan qui semble « flâner » dans un quartier qu'il ne fréquente pas habituellement, alors qu'autour de nous tout le monde travaille. Il est aussi musulman, et la consommation d'alcool fréquente dans cet espace semble le gêner. Ainsi, lorsque le chef du syndicat de transport présent sur le rond-point me tend du gin, L. se met en retrait. Si L. est assistant de recherche, c'est du fait de sa proximité linguistique avec le terrain, mais ce choix ne prend pas en compte sa distance sociale.

Trois conclusions s'imposent à l'issue de cette première journée. Tout d'abord, mon positionnement et mon comportement « hors norme » dans cet espace - « out of place » selon les termes de Tim Cresswell (1996) - me sont alors particulièrement apparus lorsque l'assistant de recherche de notre groupe n'était pas visible, par exemple lorsque nous nous sommes séparés pour nous diriger vers Mapo Hall. Travailler avec un assistant de recherche n'a alors rien de neutre, ni sur l'insertion dans un terrain, ni dans nos relations de travail et enfin pas non plus dans la production de données empiriques. Tout guide et connaisseur de la langue yoruba qu'il soit, L. est avant tout un étudiant ${ }^{12}$. Nos diverses positions sociales ont des conséquences notables sur la triple relation entre chercheur-se/assistant·e de recherche et enquêtée's. Avec L., nous n'avons pas échangé sur nos relations de travail et je regrette qu'il ne puisse ici contribuer à cette analyse. 
Figure 2. Rond-point de Beere, statue d'Oluyole, Ibadan

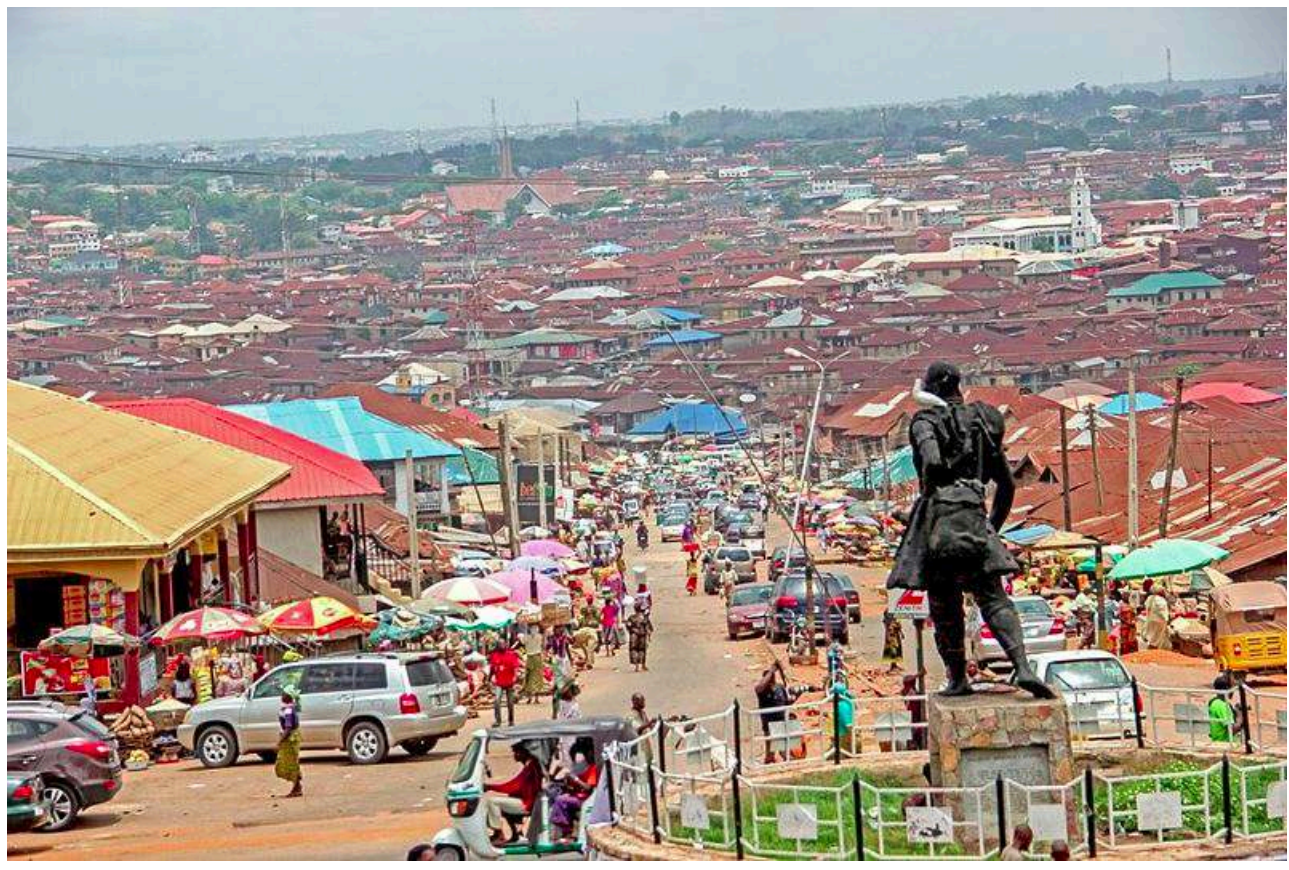

Source : Kaizenify, 2017, CC-BY-SA

13 Si la littérature anglophone est abondante sur les relations de domination et les biais liés à l'invisibilisation des assistantees de recherche dans les résultats de recherche (Turner, 2010), la littérature francophone ne s'est pas encore véritablement emparée de cette question (Kesseler, 2016). Leur rôle multiple, pouvant aller du simple accompagnement, à la production de données empiriques en passant par la traduction, les rend indispensables dans certains contextes de recherche, mais ils/elles demeurent encore souvent invisibilisée's ou reléguée's au simple remerciement en tête d'article.

14 Notre présence plusieurs jours d'affilée, à différents moments de la journée, a permis de voir se renforcer ou au moins se modifier notre rapport aux terrains et aux groupes de recherche, et la manière dont nous y étions perçue's.

\section{PRENDRE PLACE SUR UN TERRAIN}

\section{POUVOIR RESTER SUR UN TERRAIN}

Une fois entrées dans le terrain, après en avoir franchi les seuils successifs avec succès, il s'agissait de pouvoir rester durablement dans des espaces fonctionnels, animés, où se déploient des activités diverses, et au sein desquels notre passivité apparente, liée à notre statut d'observatrices, détonnait. Ainsi, l'enjeu était de légitimer notre présence. Pour cela, nous avons essayé de prendre part aux activités, à la vie du lieu, de quelque manière que ce soit, en participant à la préparation de l'amala ${ }^{13}$, au sein de l'agboole ${ }^{14} \mathrm{de}$ la famille Agate, par exemple. Dans les espaces publics marchands comme le mall et Oje Market, cela a pu passer par des pratiques, feintes ou non, de consommation. Au mall particulièrement, nous avons observé une pratique répandue parmi les usager·e's, consistant à se photographier devant les enseignes, se mettant en scène dans une position de consommateurrice's quand bien même ils ne consommeraient pas 
véritablement. En effet, une partie de ces boutiques a fermé, laissant parfois seulement quelques produits en vitrine, et celles qui sont encore ouvertes semblent désertées par les cliente's. Nous avons alors pu nuancer notre approche: si, dans sa fonction première et dans les représentations, le mall est avant tout un espace dédié à la consommation, il ne l'est plus dans les faits. C'est pourquoi, dans le cadre de cet espace d'étude, légitimer notre présence est moins passé par l'activité que par l'inactivité, du moins en apparence: s'asseoir sur un banc, regarder les gens passer, téléphoner, converser, profiter de la quiétude du lieu sans cesse opposée, par ses usager·e·s, à l'agitation continuelle du marché. En d'autres termes, nous sommes passées de l'observation diffuse à l'observation participante (Gold, 2003). Outre notre participation aux activités habituelles des espaces publics, notre présence se justifiait par la pratique d'activités directement liées à la recherche. Entre Beere et Mapo Hall, cartographier collectivement l'intersection a suscité l'intérêt et la curiosité de plus d'un·e usageree, facilitant ainsi nos interactions avec ces dernierees.

Figure 3. Projet de cartographie collaborative du rond-point de Beere

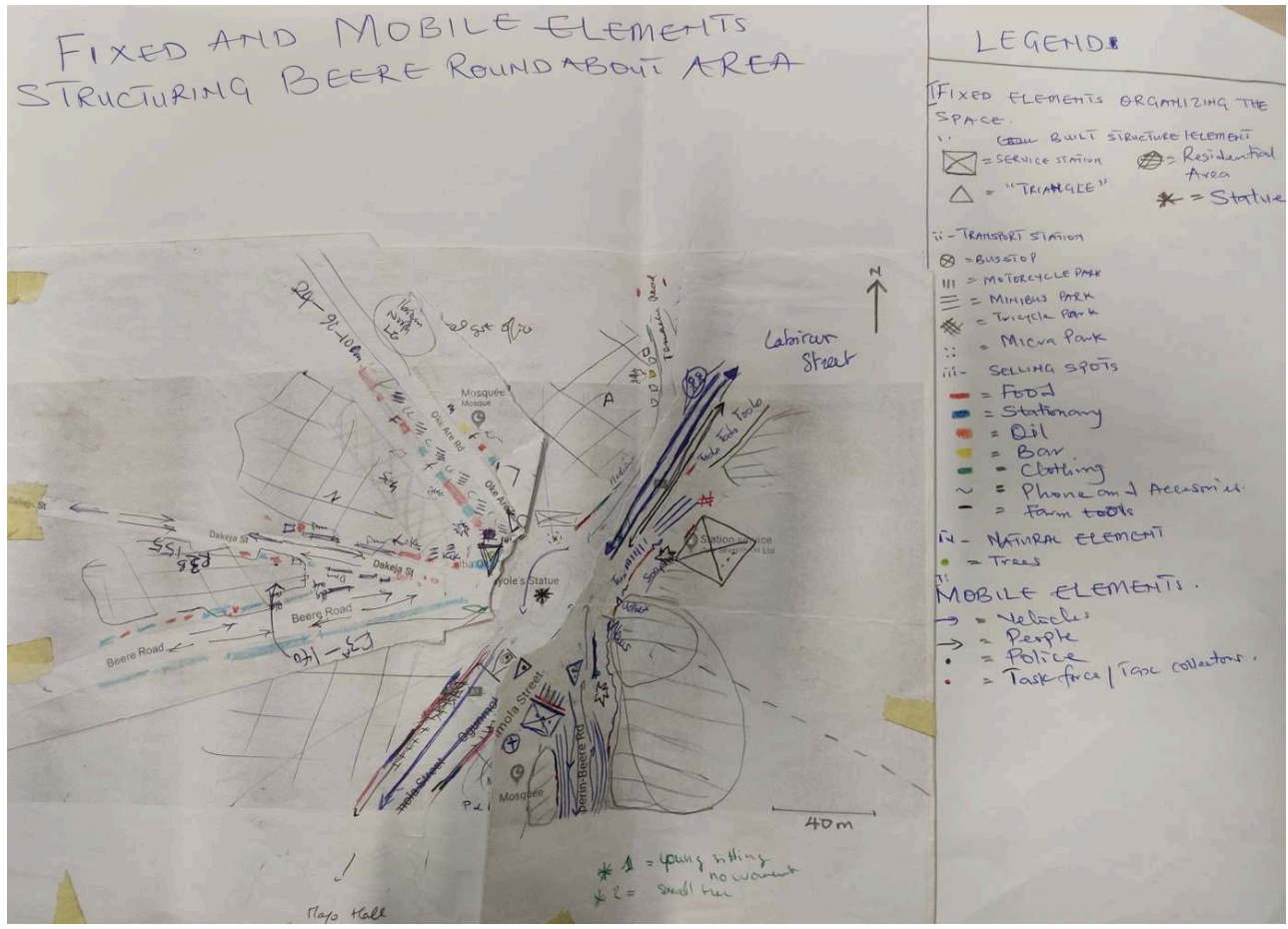

Schéma réalisé collectivement lors de la masterclass.

Le recours à la photographie, outil complémentaire de la cartographie en géographie, n'a pas toujours été possible et a donc varié en fonction des espaces d'étude. Au sein des agboole familiales par exemple, photographier les enfants était impossible pour des raisons éthiques, aussi le dessin a été privilégié, générant très rapidement un attroupement d'enfants autour de la dessinatrice. Les enfants, d'eux-mêmes, attiraient l'attention de la dessinatrice sur des lieux ou des objets de l'espace à représenter, ou prenaient le crayon. Par ailleurs, les deux familles Agate et Delesolu qui tiennent Oje Market constituent des groupes d'influence avec lesquels il a fallu négocier notre présence sur le terrain. L'identification de ces groupes d'influence ainsi que l'obtention de leur assentiment étaient des conditions nécessaires pour pouvoir rester de façon plus pérenne sur le terrain. Au rond-point de Beere, c'était avec le syndicat de transport 
qu'il fallait négocier sa place; au mall, les services de sécurité et le manager. Notre présence prolongée sur nos terrains respectifs a, à cet égard, été un révélateur des rapports de force existant entre les acteurs en présence dans ces espaces.

En dépit de la brièveté de nos terrains, l'enjeu était donc d'arriver à nous intégrer à l'espace, ce que nous avons fait en abandonnant ponctuellement notre position d'observatrices pour prendre part à des activités avec les usagere-s des espaces publics, et en justifiant et négociant notre présence auprès de ceux que nous identifiions comme des acteurs influents, garants de l'accès aux différents espaces.

\section{CE QUE LA DISTANCe (DÉ)MONTRE}

Lors de cette masterclass, nous avons considéré a priori qu'il y avait un intérêt dans l'observation directe et contextualisée d'espaces publics. En effet, l'observation permet de capter des comportements au moment même où ils se produisent, sans passer par le discours. Toutefois, nous nous rendons rapidement compte qu'elle induit des modifications potentielles des comportements du fait de notre simple présence. Ainsi, le rôle tant occupé qu'assigné dans la situation donnée conditionne l'observation et contraint, en partie du moins, le recueil d'informations. Il nous faut nous rendre à l'évidence : il ne s'agit pas d'observer une situation «telle qu'elle serait s'il n'y avait personne pour l'observer » (Vieillard-Baron, $2005: 425)$.

\section{Encadré 3 : Récit de deux expériences à Agodi Gate : visibilité et invisibilité de} la distance physique

Étudiante en géographie à l'ENS, j'étudie également l'urbanisme et l'aménagement du territoire à l'université Paris 1 Panthéon - Sorbonne.

À mon arrivée à Agodi Gate, je suis frappée par une double densité : celle du trafic routier collectif et privé, et celle des activités diverses plus ou moins annexes au transport qui prennent place le long des axes et aux abords du croisement, qui me surprend, étant habituée à une séparation des fonctions plus marquée. Agodi Gate est une gare routière très fréquentée qui fut longtemps l'une des principales portes d'entrée dans la ville. Après une première journée passée à arpenter le croisement, les stands des marchandees de rue et le marché des pièces détachées pour automobiles (spare-part market), l'organisation et le fonctionnement m'apparaissent toujours complexes et ma position d'observatrice in situ ne me satisfait pas. Remarquant un balcon au premier étage d'un magasin de pneus, j'envisage d'y monter : la distance offerte par la hauteur me permettra certainement d'observer plus finement le déploiement des activités routières et marchandes. Je demande l'accès au balcon à l'homme qui tient le magasin; celui-ci me l'accorde. Du balcon, je peux éprouver certaines de mes hypothèses sur l'organisation du croisement : il se structure autour des moyens de transport collectif qui occupent l'espace de la route dans l'attente de passager'e-s; les départs se font suivant l'ordre d'arrivée des véhicules ; chaque moyen de transport a une place bien déterminée : au centre se trouvent les okadas, des taxi-motos collectifs ; sur les différents embranchements prennent place les kekes, tricycles couverts pouvant transporter entre un et quatre passagerees, et les danfos, des taxis collectifs, plus loin les minibus; et enfin aux extrémités de la principale route 
traversante, les bus régionaux. C'est un espace dont la cohérence provient du transport, l'ancienne fonction de porte est ainsi plus clairement identifiable. Agodi Gate, comme nœud de transport, n'est pas seulement un point de départ et d'arrivée, c'est aussi un point de l'espace où se nouent les fils de multiples réseaux.

L'impression de " ne pas participer ", d'être à distance, est toutefois rapidement mise à mal. Un sentiment d'étrangeté, de ne pas être à ma place m'envahit. J'observe des gens s'affairer, des transports collectifs s'arrêter et repartir ; je suis sur le balcon d'un garagiste pour des raisons inhabituelles. Si lors de ma «flânerie observante ", j'avais pu être perçue comme une cliente potentielle, depuis le balcon, je me place dans une posture assumée d'observatrice, carnet et stylo à la main : quelle peut légitimement être la place d'une observatrice dans une gare routière?

Cette prise de distance sur le balcon me rend inévitablement visible : positionnée en surplomb avec mon carnet, je ne suis plus une passante, qui était déjà pourtant doublement visible, du fait de mon genre et de ma couleur de peau. J'attire les regards des piéton'ne's et vendeur'se's qui me font des signes pour attirer mon attention. Vers 18 heures, toujours au balcon, la nuit tombant, les activités marchandes commencent à cesser. Je remarque un regroupement autour de ce qui me semble être un puits, encadré par des bancs. Des jeunes hommes puisent de l'eau et la proposent à la vente à qui veut se laver les pieds. Soudain, cinq d'entre eux se tournent vers le balcon, me font des signes, se mettent debout sur les bancs et se mettent à danser, accompagnés par la musique d'un vendeur à proximité. D'autres s'approchent, les encouragent par des acclamations et semblent continuer à vouloir attirer mon attention. La hauteur, que j'avais naïvement imaginée comme un point de recueil d'informations plus neutres, se révèle finalement être un vecteur de visibilisation de ma présence et modifie le déroulement de la fin de la journée, du moins pour ce groupe d'individus.

Le lendemain, en début d'après-midi, un vent très fort se met à souffler, annonciateur de la pluie qui s'abat soudainement. Avec la pluie, le rythme de la place s'accélère, les okadas et kekes trouvent plus de passagers, le centre de la place se vide progressivement, les marchand·e's sur les côtés ouvrent de grands parapluies pour protéger leurs biens. Avec T., nous cherchons un abri, et pensons au balcon qui nous avait offert une vue surplombante la veille : il était en renfoncement dans la façade et offrait une protection. Nous y montons donc. Cette fois, bien que dans la même situation, personne ne semble prêter attention à notre présence, tout le monde est trop occupé à se protéger de la pluie. Lorsqu'elle cesse, le carrefour s'anime de nouveau, les okadas reprennent leur position d'attente de passagere's au centre du croisement, les parapluies des marchande's se referment. Ma situation d'observatrice, qui la veille m'avait semblé influer sur certains comportements, est dans ce cas à peine remarquée. 
Figure 4. Activités marchandes, de transport et de production à Agodi Gate, Ibadan

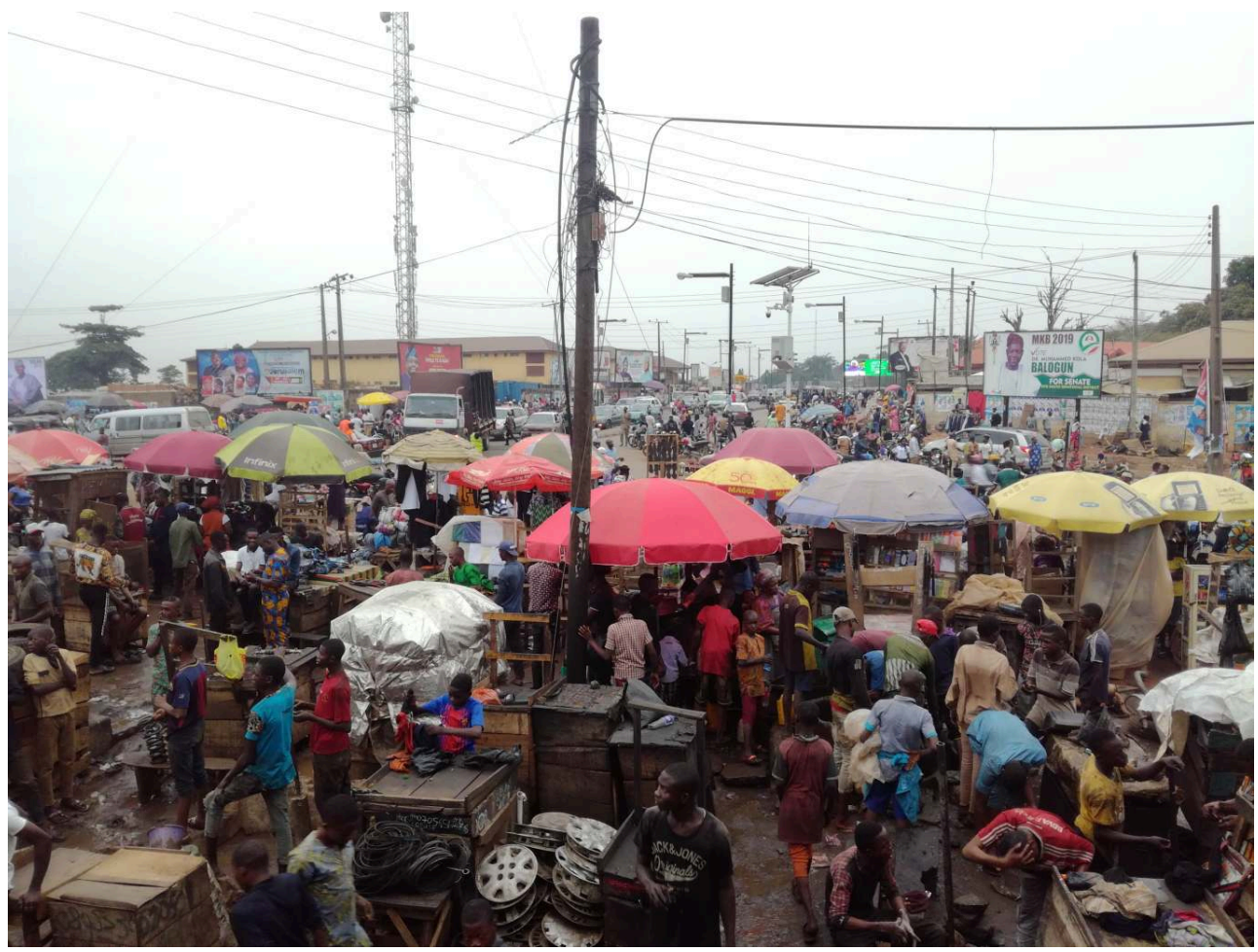

Source : autrices, 26 février 2019, vers 11h.

Figure 5. L'arrivée de la pluie remodèle les activités à Agodi Gate, Ibadan

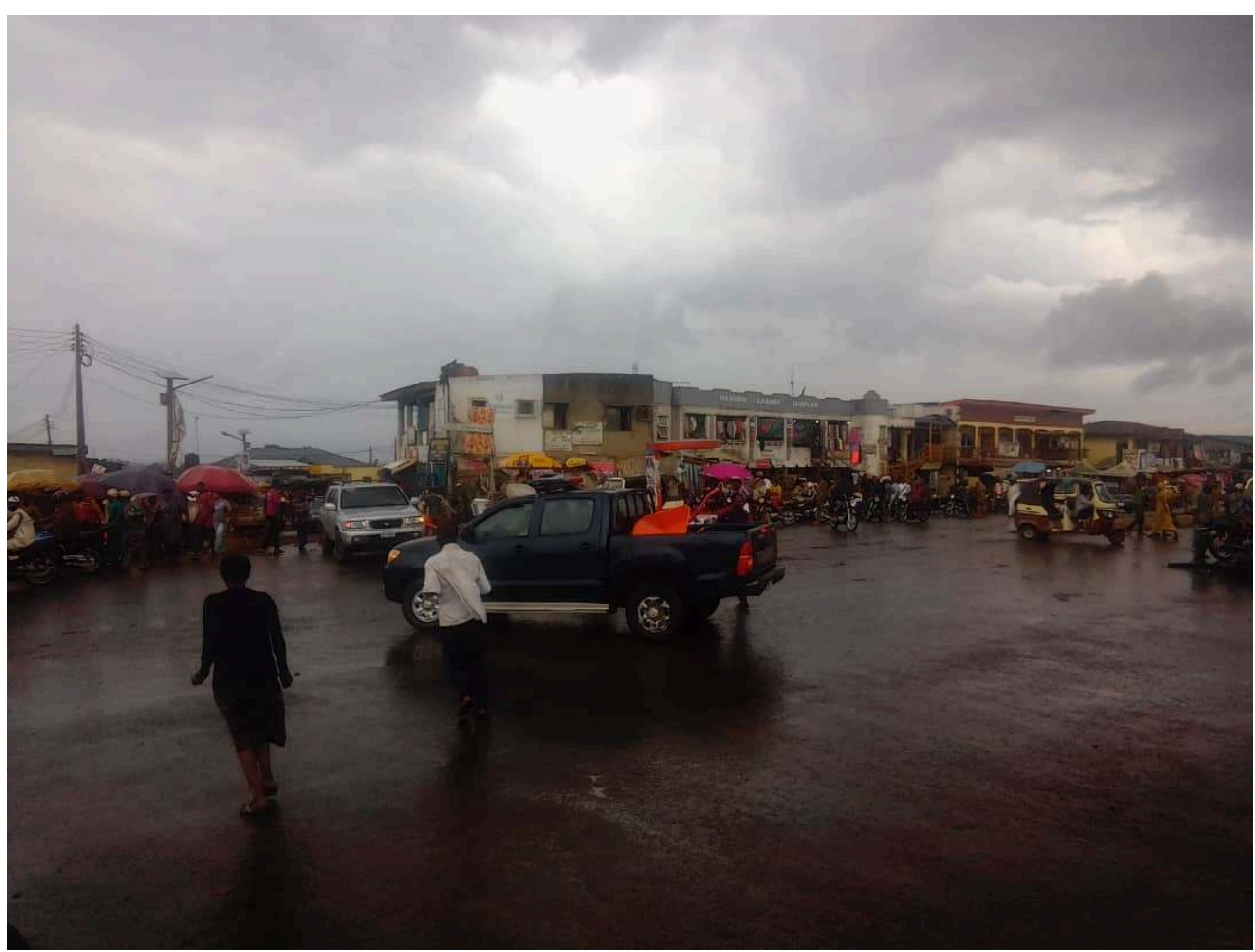

Sources : autrices, 27 février 2019, vers 17h30. 
De plus, la présence des assistantees de recherche a pu exercer une influence sur le recueil des données et renforcer une distanciation du terrain. L'anglais est une des langues officielles du Nigeria, mais beaucoup de personnes s'adressaient à nous en yoruba. Il nous est parfois arrivé que les assistante-s de recherche refusent de traduire certaines informations, lorsqu'ils considéraient subjectivement qu'elles n'étaient pas appropriées et/ou n'apporteraient rien à la compréhension de la question de recherche de la masterclass et de chaque terrain. Ce fut notamment le cas à Agodi Gate : un homme donne à l'une d'entre nous une petite pochette en papier contenant vraisemblablement une poudre et l'enjoint à la boire mélangée à de l'eau ou du jus d'orange, « it will heal all your problems $»^{15}$ dit-il. Il poursuit en yoruba, s'adressant directement à T., l'assistant de recherche du groupe. À la fin de l'interaction, T. refuse de traduire et dit que c'était "not interesting and useless for the research ${ }^{16}$. L'explication ne viendra que le dernier jour de la masterclass : il s'agissait de viagra, ce que T. avait jugé indécent de raconter sur le moment. Une expérience similaire fut vécue au rond-point de Beere, où l'assistant de recherche a refusé de traduire ce que des enceintes diffusaient, sous prétexte qu'il s'agissait simplement d'une liste de médicaments.

20 À cette distance à la fois géographique (être du lieu) et linguistique (parler yoruba sans la médiation d'un traducteur), s'ajoutent des distances culturelles et sociales. La posture d'étrangères induit une mise à distance pratique, puisqu'elle permet notamment de poser des questions naïves et suscite des réactions diverses. Ainsi au rond-point de Beere, le chef du syndicat de transport explique à l'une d'entre nous que son rôle de chef lui revenait de droit, de fait de son grand âge. Lui demandant son âge, il rit et répond « 20 ans ». Surjouant une posture naïve et extérieure, l'étudiante lui répond en avoir 25 , et qu'étant plus vieille, le rôle de cheffe du syndicat devrait lui revenir. L'homme a de nouveau ri, un peu jaune, et répondu "but you are a woman $»^{17}$. L'importance du genre et de l'âge dans les syndicats de transport est ainsi apparue et a permis d'initier une discussion sur l'existence de jeunes conductrices.

De prime abord, il est aisé de considérer les espaces étudiés, leur fonctionnement et les échanges qui s'y développent comme nous étant étrangers. Mais rapidement, la présence sur le terrain oblige à surmonter cette extériorité pour se penser comme présentes et inductrices de potentiels troubles.

\section{DES TERRAINS DANS UN TERRAIN : DES TERRAINS À TEMPORALITÉ MULTIPLES}

\section{AU RYTHME DU TERRAIN}

Nous avons spontanément distingué dans nos terrains deux formes de temporalités parfois complémentaires voire poreuses : la quotidienneté et l'événement.

La majorité des activités des usager'e's s'inscrit dans une répétition et joue par là le rôle de repères temporels. Cela implique une pratique de terrain qui s'inscrit tant bien que mal dans cette trame. L'heure du déjeuner entrâne par exemple un pic d'affluence dans les espaces dédiés aux commerces de bouche. Cette réitération des activités à un horaire donné de la journée constitue également lors de l'enquête un repère permettant de caractériser plus finement les espaces et d'appréhender leur fonctionnement. Il est ainsi possible de considérer les conditions dans lesquelles le 
déjeuner prend place, de voir qui prend en charge la préparation des repas, d'observer les mouvements urbains que le déjeuner entraîne systématiquement, etc. Ceci a été particulièrement perceptible dans le cas d'Oje Market (Encadré 4). Bien que nos expériences de terrain aient été brèves, nous avons pu apercevoir l'inscription de leurs usagere's dans ce rythme propre aux habitudes. Une telle organisation des activités, qui est peut-être plus aisée à remarquer pour qui n'en est pas coutumier, n'est pas parfaitement linéaire et comporte des temps forts ainsi que des temps faibles. Pics d'affluences et moments d'animations peuvent y être observés, ainsi que des moments plus calmes où les espaces se vident de leurs usagere's et voient leurs activités principales cesser, comme cela a pu être le cas lors de l'averse à Agodi Gate (Encadré 3). Des variations physiologiques, climatiques et sociales peuvent donc être observées dans le temps sur un même espace; elles répondent à une dynamique qui lui est propre (Sansot, 1973).

Les variations inhérentes à cette dynamique se distinguent de réels moments de perturbation lors desquels l'activité des espaces est ponctuellement altérée, qui relèvent pour leur part de l'événementiel. La pluie peut, par exemple, entraîner des déplacements massifs vers les magasins en rez-de-chaussée situés près d'Agodi Gate ou vers le mall, où il est plus facile de s'abriter. Une telle perturbation recompose le terrain en modifiant la répartition de ses usagers dans l'espace et en conduisant à la cessation de certaines activités. Elle impose également de renégocier ponctuellement sa place sur le terrain, comme ce fut le cas dans l'agboole des de la famille Delesolu où les femmes et les hommes ne se sont pas abrités de la pluie du même côté du bâtiment principal, et où le groupe de recherche mixte a donc dû se positionner dans un espace situé entre ces deux pôles.

Attirant l'attention des usager-e's de nos terrains et les détournant de leurs activités habituelles, nous introduisions nous-mêmes une part d'événementiel dans la quotidienneté. La réaction des usageres de l'agboole de la famille Agate face à l'intrusion d'un binôme constitué d'une étudiante française et d'un étudiant nigérian, et les activités différenciées qui ont été proposées à ces deux arrivant·e-s, à savoir le jeu pour l'homme et la cuisine pour la femme, n'ont par exemple que mieux permis de saisir par la suite la répartition genrée des activités dans cet espace (Encadré 4). Enfin, les effets de la présence d'une jeune femme blanche ne sont pas les mêmes selon les moments de l'enquête de terrain et demandent à être envisagés au regard des temporalités multiples au cours desquelles elle intervient. Si les événements que sa présence suscite ou auxquels elle prend part sont des révélateurs privilégiés de certains aspects des terrains étudiés, le champ restreint des possibles qui s'offre à elle pour intervenir au quotidien dans un espace est lui aussi significatif.

\section{Encadré 4 : Une journée dans l'Oje Market}

Elève en troisième année de l'ENS Lyon et en deuxième année de master d'Histoire de l'art à l'université Paris-Nanterre, mes réflexes d'historienne de l'art tiennent principalement à un besoin de représenter et à une recherche constante des lignes de force organisant le visible. Plus que le recours au dessin ou à la photographie, qui figurent déjà dans les techniques de terrain couramment mobilisées en sciences humaines et sociales, ces réflexes ont surtout dirigé mon regard sur des 
caractéristiques du paysage du marché qui n'étaient pas celles mises en avant par les usagere's interrogée's ou les autres membres du groupe de recherche.

Petit à petit, le rythme de la journée au marché semble se dessiner. Les différentes personnes occupant les agboole des familles Agate et Delesolu (les plus influentes sur le marché du fait de leur inscription historique sur les lieux et de leurs propriétés foncières) vivent sur place ou arrivent tôt le matin. Ce moment est calme et silencieux, dédié aux diverses préparations : celle des enfants pour l'école, de la mise en place des étals des marchand·e's, de la préparation par des groupes de femmes du poisson fumé dans l'agboole de la famille Delesolu ou de l'amala qui sera vendue le midi dans l'agboole de la famille Agate. La préparation est donc une activité essentiellement féminine, et les espaces sont alors principalement occupés par des femmes ou des personnes âgées. Les menuisiers et les tailleuses travaillent déjà et annoncent de par leurs positions respectives une répartition genrée des activités qui restera visible tout au long de la journée. En effet, si les mouvements du soleil redessinent progressivement les contours des zones ombragées dans lesquelles chacune évolue et contraignent les occupant·e-s des agboole à se déplacer en emportant avec eux/elles divers éléments de mobilier, cela n'affecte pas réellement l'occupation des lieux et les regroupements genrés se conservent dans leur ensemble.

Le midi apporte ses odeurs des repas ainsi que les personnes venues déjeuner. Le marché devient alors un lieu d'échanges commerciaux et verbaux intenses. Cette animation subsiste même après le départ des personnes venues se restaurer mais n'affecte pas pour autant l'organisation des agboole. C'est surtout en fin d'aprèsmidi que le marché se trouve sensiblement modifié dans ses composantes. La sortie des classes entraîne l'arrivée des enfants dans l'agboole des Delesolu, où se trouve une école primaire, ainsi que dans le marché. Ces espaces sont alors plus peuplés et bruyants que dans le reste de la journée. Quelques temps après, l'accès aux réservoirs d'eau situés dans l'agboole des Delesolu est rendu possible, ce qui conduit également de nombreuses personnes à s'y rendre. Par ailleurs, il s'agit du moment où les marchande's commencent à préparer les plats qui seront vendus au cours du marché nocturne. Les activités des menuisiers et des tailleuses cessent, tandis que les tenancières du point de vente d'amala rangent leur matériel et ferment boutique. Le marché aux herbes dans lequel travaillent surtout des femmes et qui marque l'entrée dans l'agboole des Delesolu se vide également. L'activité atteint au contraire un pic dans la salle de jeux de l'agboole des Agate, où des hommes se réunissent pour boire et regarder la télévision. Le soir correspond ainsi à un moment de transition pour les espaces du marché et des agboole qui se traduit par un changement dans leurs usagere's ainsi que dans leurs fonctions. Les femmes qui se trouvaient dans les agboole laissent notamment place à une population masculine devenue dominante.

Lorsque la nuit tombe, les agboole se vident, seuls y subsistent quelques jeunes hommes en train de fumer. L'animation du marché se concentre dans la rue, où le commerce reprend à la lueur des bougies ou des lampes électriques, accompagné de nouvelles odeurs de nourriture. Les agboole ne semblent pas nécessairement être des lieux de résidence, en témoigne celle des Agate dans laquelle bien peu de personnes restent dormir et où l'habitation principale sert à la fois de résidence 
pour le doyen et de réserve pour certain·e's marchand·e's qui y entreposent leurs marchandises pour la nuit.

Le marché est un espace dans lequel les activités laborieuses, ludiques et scolaires rythment un quotidien partagé. J'ai donc dû m'insérer dans ce quotidien, profitant des possibilités qu'il offrait et m'accommodant des limites qu'il m'imposait. Il était beaucoup plus facile de négocier ma place sur le terrain le matin, quand l'activité n'était pas trop intense, que les femmes étaient majoritairement présentes et que certaines me reconnaissaient d'un jour sur l'autre, tandis que le repas du midi ou la sortie des classes renforçaient nettement ma visibilité tout en me poussant à prendre part aux activités collectives. Surtout, j'étais ouvertement invitée à quitter les agboole le soir avec le reste de ses occupant·es de la journée, et les usager.e's de ces espaces comme certain'e's membres de mon groupe de recherche ne m'ont pas permis de pénétrer dans la salle de jeux exclusivement masculine, ne m’y jugeant pas à ma place ou pas en sécurité.

Figure 6. Panneau marquant l'entrée dans l'agboole de la famille Agate à Oje Market, Ibadan

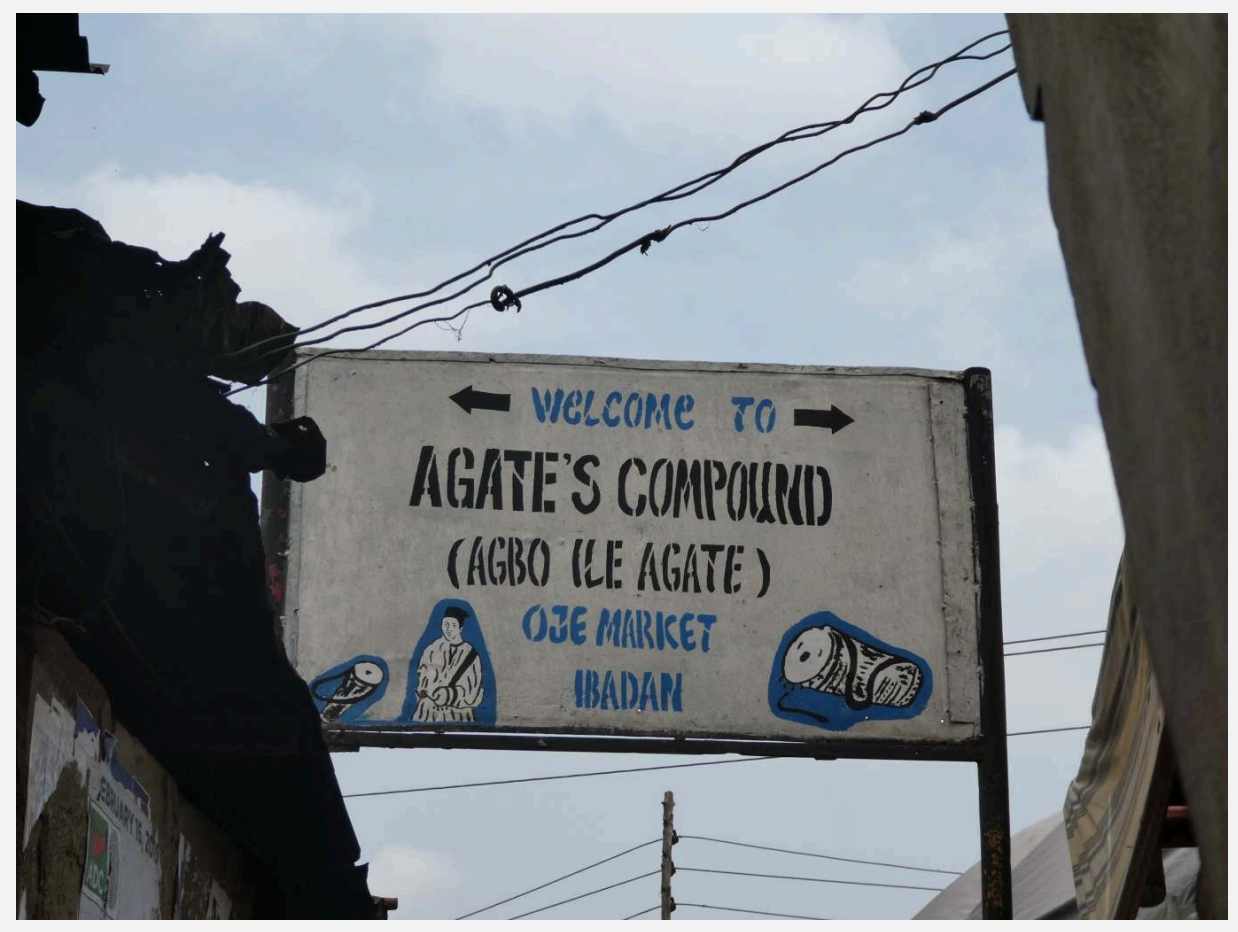

Source : autrices, 27 février 2019, vers 12h15. 
Figure 7. Vue du lieu de préparation et de distribution de l'amala au centre de l'agboole de la famille Agate à Oje Market, Ibadan

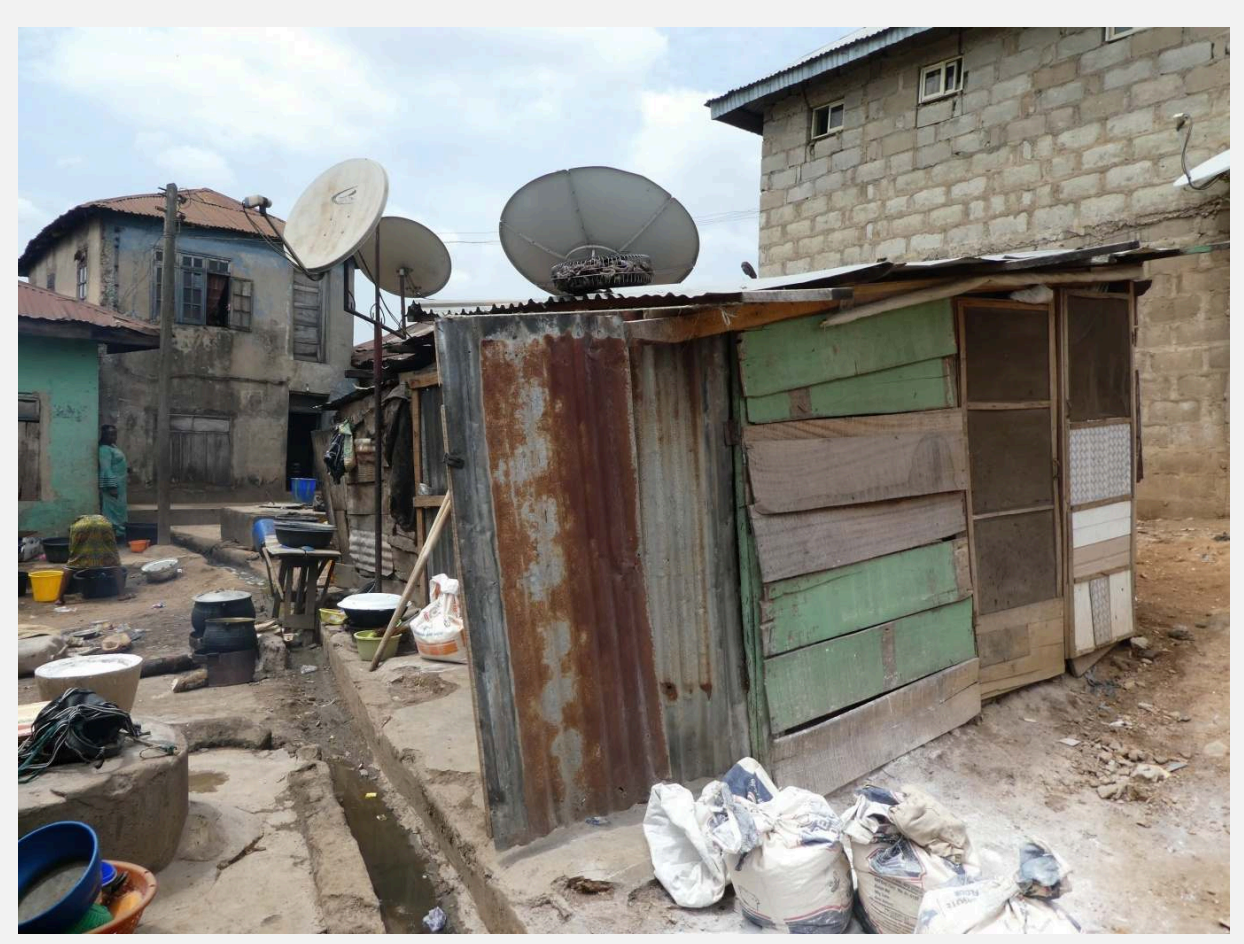

Source : autrices, 27 février 2019, vers 12h.

Figure 8. Vue de l'enclos du crocodile sacré de la famille Delesolu et de l'amandier, tous deux au centre de l'agboole à Oje Market, Ibadan

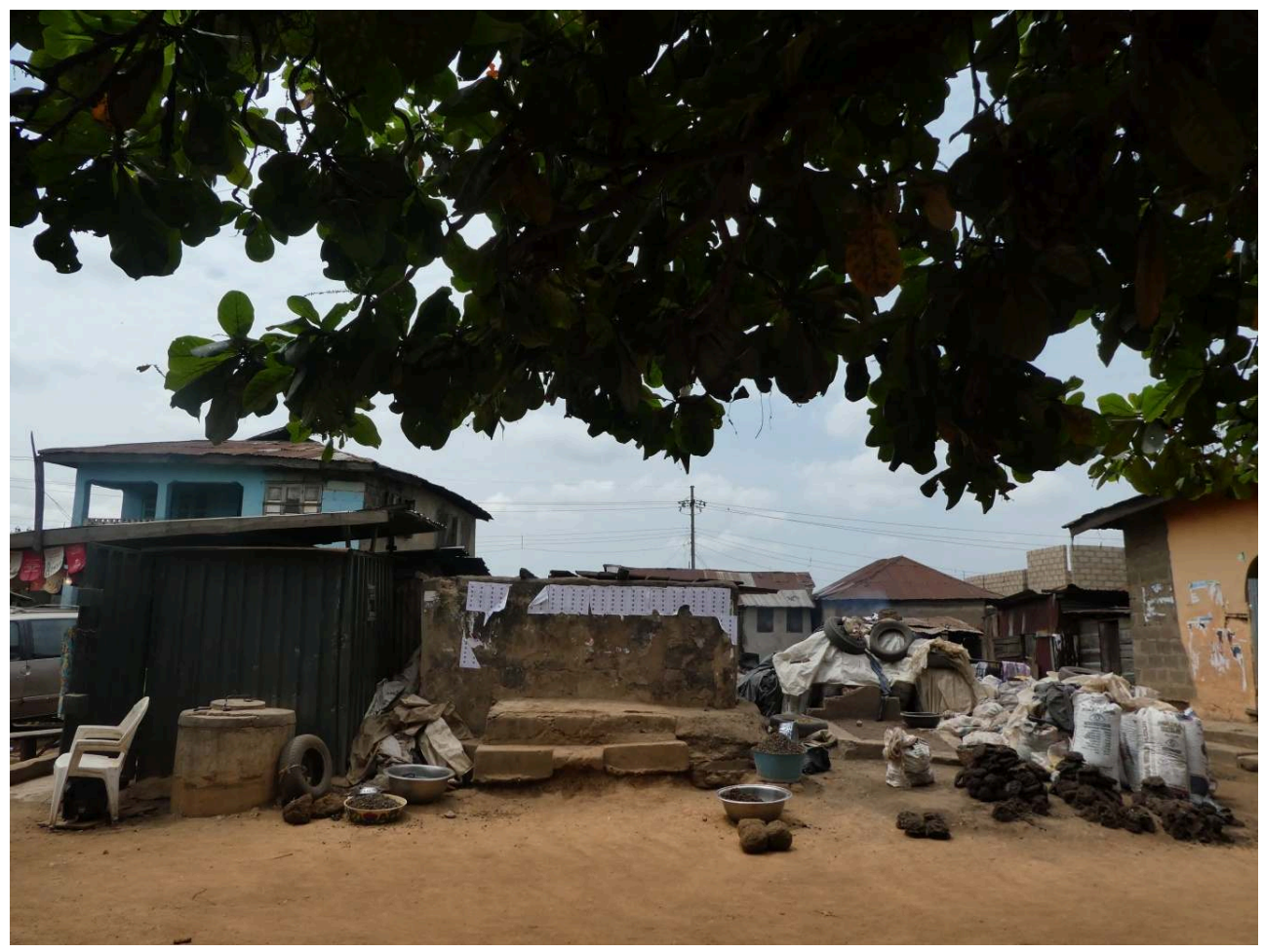

Sources : autrices, 27 février 2019, vers 12h. 


\section{QUITTER LE TERRAIN} permettant d'observer les espaces à différents moments de la journée. Cette présence nocturne a été interrompue différemment en fonction des terrains, ce qui nous renseigne sur les changements qui s'y produisent une fois la nuit tombée. Certaines sorties de terrain ont ainsi simplement consisté à imiter les usager-e's des espaces lors de leur fermeture. Il en va ainsi du mall dont les usagere's sont évacuée's à un horaire précis, ou bien des agboole progressivement délaissés alors que la rue attire leurs usager-e's. Ces mouvements de l'ensemble des occupant-e's d'un espace se sont effectués dans une forme de continuité avec les activités quotidiennes, la sortie du terrain, conséquente de sa fermeture, s'opérant dès lors sans heurt. Ce n'est pas le cas d'autres sorties de terrain, qui ont été imposées par les circonstances. À Beere par exemple, différents facteurs ont conclu abruptement le terrain nocturne: l'assistant de recherche a considéré que la nuit accroissait la dangerosité de l'espace, ce qui ne permettait plus de poursuivre l'étude dans des conditions de sûreté suffisantes; le chef $\mathrm{du}$ syndicat de transport a insisté auprès du groupe de recherche pour qu'ils/elles quittent les lieux. Ce départ, comme celui effectué à Agodi Gate, s'explique également par un climat politique tendu puisqu'il s'agissait du jour de l'annonce des résultats des élections générales nigérianes ${ }^{18}$. Ces sorties de terrain forcées soulignent les temporalités et les usages différents qui peuvent caractériser un même espace ainsi que les écarts dans la manière dont il peut être perçu et donc étudié le jour et la nuit.

\section{CONCLUSION}

Si cet article n'a pas la prétention de proposer une compréhension fine des quatre espaces publics que nous avons étudiés, ce que la brièveté de nos présences sur le terrain rendrait de toute évidence impossible, l'expérience de recherche in situ est riche et révélatrice des différences de positionnements qu'implique une recherche en groupe dans des contextes d'espaces publics à Ibadan. L'expérience de recherche collective, si elle peut engendrer des difficultés et des incompréhensions, permet de mettre en évidence la dimension éminemment relationnelle de tout terrain dans la mesure où elle permet de confronter différentes réceptions de et insertions dans ce terrain.

Cependant, la spécificité de ce contexte ne doit pas empêcher toute démarche comparative avec d'autres espaces publics européens ou africains que nous avons pu étudier par ailleurs. En effet, cette expérience a également pu mettre en lumière des similarités avec nos expériences de recherche précédentes ou même nos pratiques plus quotidiennes des espaces publics. Ainsi le caractère essentiellement masculin de certains espaces ou temps publics pose les mêmes problèmes dans des villes européennes que ceux soulevés dans le cas de nos terrains à Beere et Agodi Gate (Rose, 1993; Lieber, 2008). Il ne s'agirait donc pas ici de surestimer une quelconque exceptionnalité culturelle du contexte nigérian. Il serait à cet égard particulièrement stimulant de mettre en place des expériences de terrain de ce type dans d'autres contextes. Plus particulièrement, pour prolonger cette masterclass et contrebalancer certains déséquilibres notés dans le présent article, il pourrait par exemple être pertinent d'inviter les participant-e's nigérian'e's à venir réaliser des terrains à Paris. 


\section{BIBLIOGRAPHIE}

CATTAN N., LEROY S. (2010), « La ville négociée : les homosexuel(le)s dans l'espace public parisien », Cahiers de géographie du Québec, vol. 54, no. 151, pp. 9-24.

CRENSHAW K. (1991), « Mapping the Margins: Intersectionality, Identity Politics, and Violence against Women of Color ", Stanford Law Review, vol. 43, no. 6, pp. 1241-1299.

CRESSWELL T. (1996), In place/out of place: geography, ideology, and transgression, Minneapolis, University of Minnesota Press.

ÇINGI KOCADOST F. (2017), « Le positionnement intersectionnel comme pratique de recherche : faire avec les dynamiques de pouvoir entre femmes ", Les cahiers du CEDREF. Centre d'enseignement, d'études et de recherches pour les études féministes, no. 21, p. 17-50.

DIKEÇ M. (2010), « Colonial Minds, Postcolonial Places », Antipode, no. 42, pp. 801-805.

DUPONT L. (2014), « Terrain, réflexivité et auto-ethnographie en géographie » in Calbérac Y., Volvey A., Géographie et Cultures, no. 89-90, pp. 93-109.

GOLD R. (2003[1958]), « Jeux de rôle sur le terrain. Observation et participation dans l'enquête sociologique », in Céfaï D., L'enquête de terrain, Paris, La Découverte, pp. 340-349.

GRANGER G. (2004), « La spécificité des actes humains », Espace Temps, vol. 84, no. 1, pp. 51-61.

HANCOCK C. (2007), « "Délivrez-nous de l'exotisme" : quelques réflexions sur des impensés de la recherche géographique sur les Suds (et les Nords) ", Autrepart, vol. 41, no. 1, pp. 69-81.

KESSELER S. (2016), «1. Être et avoir un assistant de recherche. Réflexion méthodologique sur le positionnement social de l'assistant » in Lafay M., Regards scientifiques sur l'Afrique depuis les Indépendances, Paris, Editions Karthala, pp. 33-57.

LIEBER M. (2008), « Chapitre 6 / De la peur assignée aux tactiques d'évitement », Genre, violences et espaces publics, Paris, Presses de Sciences Po, pp. 233-62.

NAVEZ-BOUCHANINE F. (2001), « De l'espace public occidental aux espaces publics non occidentaux », Villes en Parallèle, vol. 32, no.1, pp. 120-34.

ROSE G. (1993), Feminism and Geography: The Limits of Geographical Knowledge, Cambridge, Polity Press.

SANSOT P. (1973), Poétique de la ville, Paris, Klincksieck.

STAEHELI L., MITCHELL D. (2007), The People's Property? Power, Politics, and the Public, New York, Routledge.

TURNER S. (2010), « Research Note: The Silenced Assistant. Reflections of Invisible Interpreters and Research Assistants ", Asia Pacific Viewpoint, vol. 51, no. 2, pp. 206-19.

VERGNAUD C., LE GALL J. (2017), « Le stage de terrain : que transmet-on en tant qu'enseignant chercheur? Regards croisés enseignants et étudiants ", Carnets de géographes, no. 10. [En ligne : https://journals.openedition.org/cdg/1206]

VIEILLARD-BARON H. (2005), « Entre proximité et distance, quelle place pour le terrain? », Cahiers de géographie du Québec, vol. 49, no. 138, pp. 409-427.

VOLVEY A., CALBÉRAC Y., HOUSSAY-HOLZSCHUCH M. (2012), « Terrains de je. (Du) sujet (au) géographique », Annales de géographie, vol. 687-688, no. 5, pp. 441-61. 
WEBER F., BEAUD S. (2010), Guide de l'enquête de terrain, Paris, La Découverte.

ZRINSCAK G. (2010), « Enseigner le terrain en géographie », L’Information géographique, vol. 74, pp. 40-54.

\section{NOTES}

1. Nous remercions pour cela les organisateur-rice's et encadrant·e's de cette masterclass : Élodie Apard, Clémentine Chazal, Pauline Guinard, Émilie Guitard, Ismaël Maazaz, Julien Migozzi et Chrystel Oloukoï, ainsi que les participantees : Abah Danladi, Junaidu Danladi, Dorcas Popoola Oluronke, Nkwuka Nnamdi, Mariam Toyin Balogun, Ashamy Kayode, Omomayowa Abati, Edeyan Harriet Omoweh, Tobi Adewunmi, Williams Oluwaseun et l'ensemble des assistant.e.s de recherche. Cette masterclass fut possible grâce aux financements de l'EUR Translitterae, l'IFRA et le Département Géographie \& Territoires de l'ENS.

2. Il convient de nuancer les différences qui pourraient être établies entre les quatre disciplines dont nous sommes issues en précisant qu'elles relèvent toutes des sciences humaines et sociales et prennent donc pour objet d'étude des faits humains (Granger, 2004). Bien que mobilisant des systèmes conceptuels différents lors de l'interprétation de ces faits, elles accordent en cela la même primauté à leur observation et partagent donc des pratiques de terrain communes.

3. Même si les méthodes ethnographiques sont également mobilisées dans le cadre d'une distanciation nécessaire du/de la chercheur:se en « terrain » familier (Weber, Beaud, 2010).

4. Comme le montrent Camille Vergnaud et Julie Le Gall (2017), l'Ecole Normale Supérieure offre en cela une formation spécifique tournée vers l'apprentissage de la recherche par la pratique. Par ailleurs, la formation à l'ENS de Paris étant très tournée vers l'interdisciplinarité, les cadres disciplinaires de chacune d'entre nous ont probablement moins joué que dans une formation disciplinaire spécifique à l'université.

5. Si cette expérience de terrain a servi à interroger certaines dimensions de la notion d'espace public au Nigeria, nous n'avons cependant, dans cet article, ni vocation à en livrer une analyse ni à participer aux débats théoriques autour de cette notion. Le temps sur place étant limité, nous avons conçu ces quelques jours comme des terrains d'exploration permettant d'établir des questionnements de recherche. Ainsi nous avons dégagé les problématiques suivantes: Quelles sont les manifestations spatiales des relations à la fois complémentaires et compétitives entre les activités sociales et économiques au rond-point de Beere? En quoi le déclin de la fonction économique du mall d'Ibadan reconfigure-t-il les pratiques des usagere-s de cet espace public marchand? Dans quelle mesure la combinaison de multiples activités - circuler, produire, échanger - présentes à Agodi Gate en fait-elle un «territoire porte " ? Comment l'articulation de divers espaces aux niveaux d'ouverture physique et sociale variables détermine-t-elle l'organisation d'Oje Market?

6. En tant qu'étudiant·e ou chercheur'se étranger'e, travailler avec un'e assistant·e de recherche nigériane est une obligation. Nous reviendrons sur les implications de cette relation chercheur:se/assistante en cours d'article. Il convient de préciser que les assistant.e.s de recherche n'ont pas participé à la première semaine de masterclass théorique à Lagos, qu'ils et elles venaient de disciplines différentes et étaient rémunéré.e.s pour leur rôle d'assistant.e.s.

7. Traduction personnelle. Citation originale: "Such a proud display of the "fieldwork" badge of honour seems to me problematic in that it also betrays anti-Eurocentric positions as it essentially intensifies one of the hallmark consequences of Eurocentric thinking. It not only does "little to refute that sense of a world cartographically bifurcated into a theory rich west and the rest of the world construed more or less as field" (Jazeelforthcoming:5), but effectively reproduces it. » (Dikeç, 2010 : 803)

8. Mot yoruba utilisé pour désigner les blanches. 
9. Les récits sont reconstitués à partir des notes prises dans nos carnets de terrain respectifs le jour même.

10. «Tu as été suivie par deux hommes » / «Je sais mais tout va bien » / «D'accord, tu sembles savoir comment faire ».

11. «Demande-lui ce que tu veux, je traduirai » / «Merci pour la traduction, mais pose aussi tes questions si tu en as ».

12. Ce statut social particulier à Ibadan nous a par la suite permis des interactions privilégiées avec certaines vendeuses du quartier, reconnaissant en lui leur fils étudiant, tandis que les interactions avec les jeunes hommes, travaillant principalement auprès du syndicat de transport local, ont pu en être compliquées.

13. Pâte dense préparée à partir de farine d'igname et d'eau bouillante, généralement consommée avec de la soupe.

14. Terme yoruba désignant un ensemble d'habitations regroupées autour d'une cour ouverte semi-publique ou bien cette cour elle-même. Le travail sur la double dichotomie fermeture/ ouverture et propriété/publicité véhiculée par les termes yoruba agboole et ita gbangba, qui peut désigner à la fois la cour ouverte, l'espace ouvert séparant l'unité résidentielle de la rue, la rue elle-même ou encore l'espace ouvert du marché a permis de mesurer les limites et de préciser les contours des notions espace public/espace privé sur lesquelles nous nous étions appuyée's.

15. "Ça soignera tous vos problèmes"

16. «Inintéressant et inutile pour la recherche »

17. "Mais, tu es une femme »

18. Les élections générales nigérianes ont eu lieu le 23 février 2019. Elles combinaient les élections présidentielles, législatives et sénatoriales. Le scrutin devait initialement se dérouler le 16 février, mais il a été reporté le jour même au 23 février. Muhammadu Buhari (Congrès des progressistes) est réélu au premier tour face à Atiku Abubakar (parti démocratique populaire) qui conteste les résultats.

\section{RÉSUMÉS}

Cet article s'appuie sur la réalisation de brefs terrains collectifs dans différents espaces publics de la ville d'Ibadan au Nigeria. Il propose une réflexion sur l'insertion de jeunes étudiantes blanches occidentales dans ces espaces publics ainsi que dans les groupes de recherche concernés. Nous souhaitons comprendre dans quelle mesure notre positionnement a pu se révéler contraignant tout en nous renseignant sur les terrains étudiés.

This article relies on short field works realized in different public spaces in the city of Ibadan, Nigeria. It provides a reflection on young Western white female students' integration in these public spaces and in their research groups. We try to understand to what extent our position may have been both restrictive and useful for the understanding of our fieldworks.

\section{INDEX}

Mots-clés : terrain collectif, intersectionnalité, espaces publics, positionnement

Keywords : Collective Fieldwork, Intersectionality, Public Spaces, Positioning 


\section{AUTEURS}

\section{PAULINE VERGER}

Étudiante de l'École Normale Supérieure (ENS)

pauline.verger[at]ens.fr

\section{MANON LE BON}

Ancienne étudiante de l'École Normale Supérieure (ENS)

manonlebon[at]hotmail.fr

\section{MATHILDE BEAUFILS}

Ancienne étudiante de l'École Normale Supérieure (ENS)

mathilde.l.beaufils[at]gmail.com

\section{CHLOÉ VINCENT}

Étudiante de l'École Normale Supérieure (ENS)

chloe.vincent.uniparis1[at]gmail.com

\section{JUSTINE VIGNÈRES}

Étudiante de l'École Normale Supérieure (ENS)

justine.vigneres[at]ens-lyon.fr 